COPYRIGHT @ 2021 INTERNATIONAL JOURNAL OF SCIENCE DENTISTRY | AVAILABLE ONLINE http://www.periodicos.uff.br/index

\title{
TRATAMENTO CIRÚRGICO DE CANINO INCLUSO EM REGIÃO MENTUAL: RELATO DE CASO
}

Surgical Treatment of a canine impacted in Mental Region: Case Report

\section{Farley Souza Cunha}

Graduado em Odontologia pela Universidade Federal Fluminense, Niterói / RJ, Brasil.

\section{João Victor Wiechers Aieta Santoro}

Graduado em Odontologia pela Universidade Federal Fluminense, Niterói / RJ, Brasil.

\section{Emanuel Escudeiro}

Residente de Cirurgia Buco-Maxilo-Facial UNIFESO, Teresópolis / RJ, Brasil.

\section{Rafael Seabra Louro}

Professor da disciplina de Cirurgia Bucal da Universidade Federal Fluminense - UFF, Niterói / RJ, Brasil.

\section{Rodrigo Figueiredo de Brito Resende}

Professor de Cirurgia Oral Menor da Faculdade de Odontologia da Universidade Federal Fluminense, , Niterói / RJ, Brasil.

Universidade Federal Fluminense

Faculdade de Odontologia

Artigo de Relato de caso

\section{Rodrigo Figueiredo de Brito Resende}

Endereço: Rua Mario Santos Braga, 28 - Centro, Niterói - RJ, 24020-140

Telefone: (21) 997399282

E-mail: resende.r@hotmail.com 


\title{
RESUMO
}

O dente denominado incluso nada mais é que um dente retido ou impactado dentro do alvéolo dentário de maneira parcial ou completa que não conseguiu erupcionar por motivos patológicos ou simplesmente anatômicos após o período de rizogênese. $\mathrm{O}$ canino, em especial, é de suma importância sob os pontos de vista estético e funcional. O deslocamento do canino incluso ultrapassando, ou se aproximando da linha média antes da erupção dentária do mesmo, é mais frequente na mandíbula do que na maxila. Este trabalho tem por objetivo descrever o caso de um paciente de 21 anos que apresentava um canino incluso na região mentual, em posição transversal, inicialmente visualizado em uma radiografia periapical e posteriormente submetido a radiografia com a técnica de Donavan. Através de uma radiografia oclusal, foi possível observar o posicionamento do dente para a vestibular mentoniana e íntima relação do dente retido com as raízes dos incisivos centrais decíduos do paciente. Foi realizada a extração do dente retido no mento, removendo o mesmo por completo sendo feitas osteotomias e odontossecções. O paciente está em acompanhamento clínico à 1 ano após o tratamento cirúrgico.

Palavras - chave: Dente incluso; Cirurgia Oral; Imaginologia.

\begin{abstract}
The tooth inserted is nothing more than a tooth retained or impacted within the dental socket partially or completely that failed to erupt for pathological or simply anatomical reasons after the period of rhizogenesis. The canine, in particular, is of the utmost importance from the aesthetic and functional points of view. The displacement of the canine, even surpassing or approaching the midline before tooth eruption, is more frequent in the mandible than in the maxilla. This work aims to describe the case of a 21 - year - old patient who had a canine included in the mental region, in a transverse position, which was initially visualized on a periapical radiograph and later submitted to radiography with the Donavan technique. An occlusal radiograph showed the positioning of the tooth for the mental vestibular and the intima relationship of the retained tooth with the roots of the patient's central deciduous incisors. Removal of the tooth retained in the denture was performed, removing the tooth completely and osteotomies and odontosections were performed. The patient is in clinical follow-up at 1 year after the surgical treatment.
\end{abstract}

key words: Tooth included; Oral surgery; Imaginology. 


\section{INTRODUÇÃO}

A presença de um dente impactado é compreendido como insucesso em se posicionar de forma correta e harmônica na arcada dentária, estando situado parcialmente ou totalmente no interior do osso alveolar (PETERSON L. 2000). Essa impactação dentária pode promover o surgimento de lesões no órgão dentário também podendo comprometer o estado de saúde do paciente. Algumas dessas lesões ganharam destaque nas literaturas por serem mais comuns ou por terem um crescimento rápido e destruidor, como o ameloblastoma, o cisto dentígero, tumor odontogênico adenomatóide, entre outros. Outro ponto importante, é que os dentes impactados podem comprometer os dentes adjacentes através das lesões primárias ou reabsorções radiculares externas (NEVILLE et al. 2004).

$\mathrm{Na}$ atualidade, ainda é um desafio para o cirurgião dentista tratar os casos de caninos impactados, pelo fato de envolver o quesito estético e funcional desses dentes. Quando o canino inferior está proximo de sua localização de origem fisiológica, porém impossibilitado de erupcionar, é chamado de impactado,ou incluso.

A aparição de caninos impactados na mandíbula não é muito comum. Foi observado que a inclusão ou impactação dos caninos superiores inclusos ocorre numa frequência de $2,0 \%$ da população, enquanto que os inferiores em $0,02 \%$ (THILANDER, B. et al. 1973). Outro estudo feito pelo pesquisador ROHRER, A, examinando radiografias panorâmicas de 3 mil pacientes encontrou 62 caninos maxilares inclusos $(2,06 \%)$ e somente 3 caninos mandibulares inclusos $(0,01 \%)$, em uma proporção de 20:1. Isso sugere uma incidência de caninos inclusos mandibulares na população estudada variou de 0,35 a $0,44 \%$.em relação ao maxilares (ROHRER, A. 1929).

A ocorrência de caninos inclusos é multifatorial, podendo ser por condições ambientais, sistêmicas ou locais, possuindo ainda, variações etiológicas (BISHARA, S.E 1992). Um desses fatores etiológicos mais encontrados são: a perda imatura do canino decíduo(ocasionando uma perda do tamanho da arcada), comprimento anormal da coroa, traumatismo e o mau posicionamento do germe dentário (CANDEIRO, G. et al. 2009).

Na maioria dos casos, a impactação do canino inferior não apresenta sintomas clínicos, como dores e desconfortos, sendo assim, de forma assintomática (LUDLOW JB, et al 2003). Nesses casos de assintomatologia, é possível detectar, 
na maioria dos casos, um aumento de volume na mucosa vestibular ou lingual durante o exame clínico (CANDEIRO, G. et al. 2009).

Para a complementação diagnóstica é preciso dispor de um bom exame de imagem. A radiografia convencional vem sendo menos utilizada como o único exame de imagem auxiliador devido ao advento da tomografia computadorizada(VUCHKOVA, J. et al 2010). Vale ressaltar que em alguns casos é realmente necessário se fazer o uso de uma tomografia, porém em outros casos as radiografias convencionais, como as panorâmicas, as oclusais (Donavan ou submentovertix) e ultilizando a regra de Clark, podem promover um ótimo exame de imagem auxiliar no diagnóstico e no planejamento do tratamento.

\section{RELATO DE CASO}

Paciente ,N.B.C de sexo feminino , 21 anos, melanoderma, foi atendida no projeto de extenção Dente Incluso, na Faculdade Federal Fluminense (UFF) a fim de remover o canino incluso na região de mento.

Após o exame clínico e avaliação radiográfica inicial, a opção de tratamento proposta foi a remoção cirúrgica do dente impactado. Para a realização da cirurgia foi solicitado um exame radiográfico oclusal oblíquo da região ( Figura 2) e uma radiografia periapical completa (Figura 3) para auxiliar no planejamento do acesso cirúrgico, sendo diagnosticada a posição do canino incluso pela vestibular

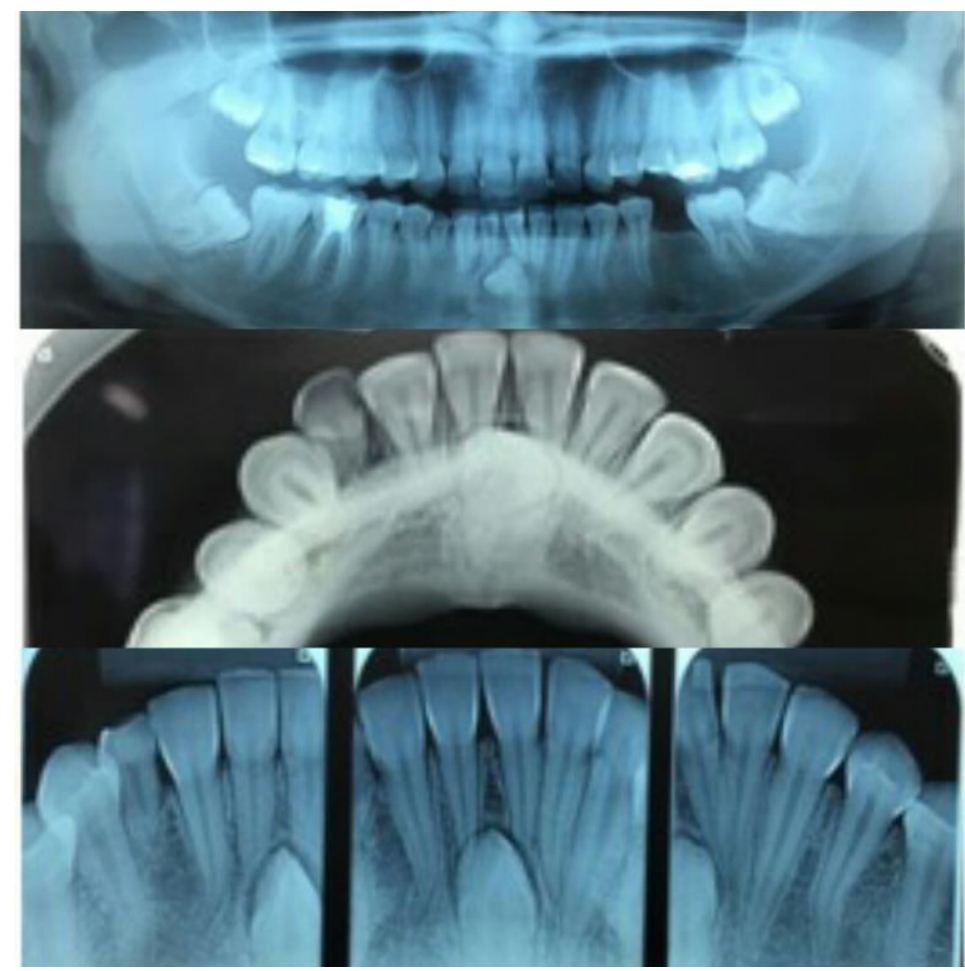

Figura1: Radiografia panorâmica sendo evidenciado o canino incluso próximo aos ápcies dentarios anteriores inferiores. Figura2: Radiografia Oclusal REVISTA FLUMINENSE DE ODONTOLOGIA - ANO XXVII - No 55 - Janeiro / Julho 2020 
(Donavan) sendo localizado possivelmente pela vestibular o canino impactado. Figura 3: Radiografia periapical da regão anterior da mandíbula, sendo possivel visualizar possivelmente o intimo contato do dente retido com os ápices dentários anteriores.

A cirurgia foi realizada sob anestesia local de lidocaína $2 \%$ com adrenalina 1:100.000 (ALPHACAINE 100, DFL, RJ, Brasil ). Após o bloqueio bilateral dos nervos mentonianos foi realizado um retalho cirúrgico do tipo envelope com lâmina de bisturi número 15 (ADVANTIVE, Sterilance, Suzhou, China) e descolamento subperiosteal com descolador de Molt para acesso ao mento (Figura $4)$.

Em seguida, foi feita a ostectomia com broca esférica número 6 e irrigação contínua de solução salina $0,9 \%$ com seringa de $20 \mathrm{ml}$, para exposição da coroa dentária (Figuras 5).

Após a ostectomia na região mentual onde localizava-se o dente 43 impactado e exposição do mesmo, foi realizada a odontossecção com auxílio de broca cirúrgica 702 acoplada à peça de alta rotação e abundante irrigação com solução salina $0,9 \%$. O dente foi cortado em 3 partes, pelo fato de seu posicionamento próximo aos ápices radiculares dos incisivos centrais inferiores, e removido com o uso de alavanca Apexo 303 (QUINELATO, Schobell Industrial Ltda, Rio Claro, SP, Brasil) (Figuras 6, 7, 8 ).

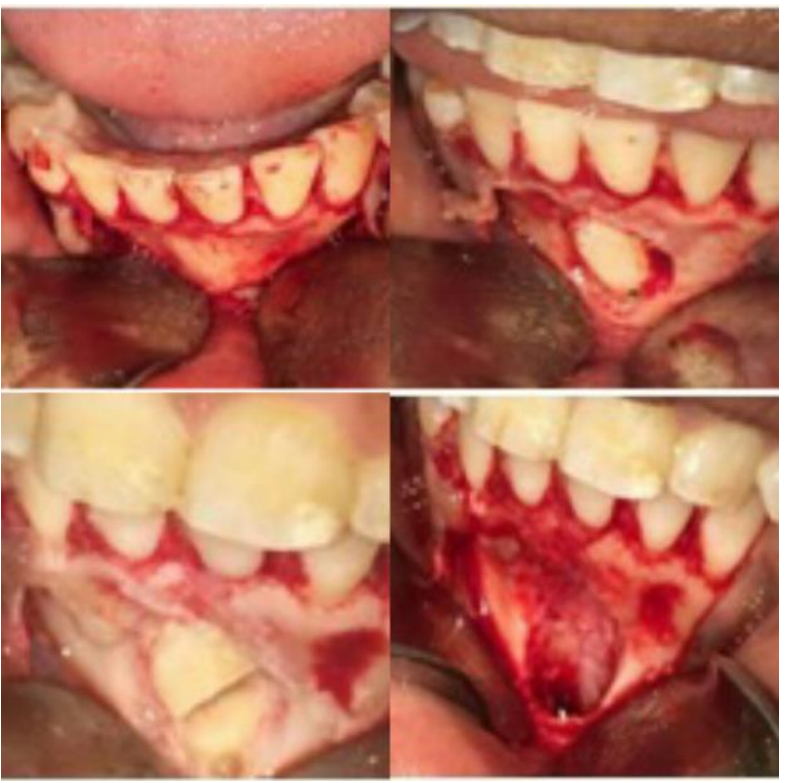

Em seguida, foi feita a curetagem do saco pericoronário, em sequência, a ferida foi lavada abundantemente e realizada sutura na musculatura interna com fio reabsorvível (VICRYL 4.0, Ethicon, Nova Jersey, EUA) e externamente com fio 
de sutura (MONONYLON 5.0, Ethicon, Nova Jersey, EUA) e fio de sutura (SEDA SILK 3.0, Ethicon, Nova Jersey, EUA ), mantida por 7 dias.(Figura 9)

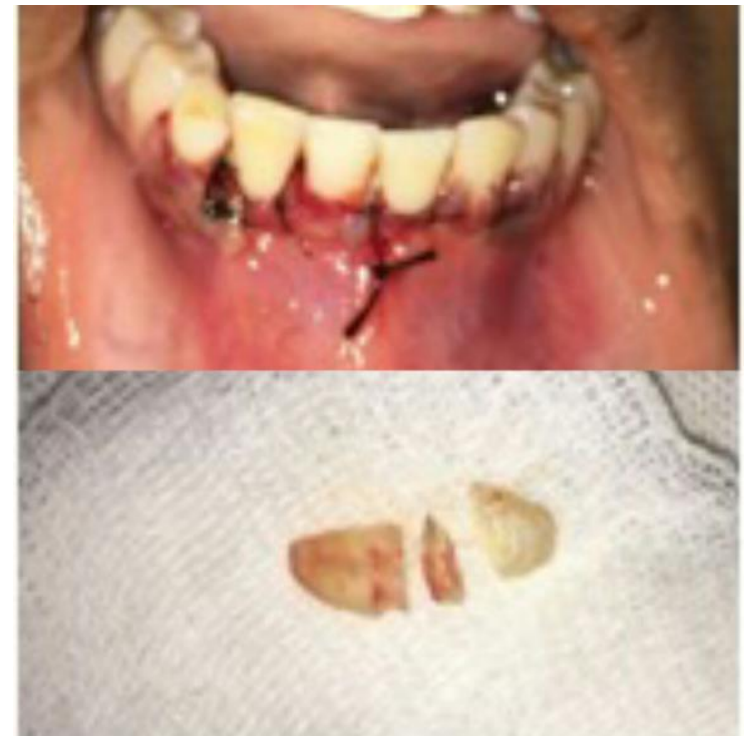

\section{DISCUSSÃO}

A ocorrência da impactação dos caninos permanentes é bastante comum. Um fato que explica essa ocorrência é o canino ser um dos ultimos dentes à serem erupcionados na arcada dentária permanente. Outro ponto a ser ressaltado é que a simples falta de espaço e a negligência no acompanhamento do crecimento ósseo e dantário da criança pode levar de uma instalação de aparelhos ortodonticos à uma extração de um dente permante( BISHARA, S.E 1992).

O caso relatado concorda com os estudos que mostram uma maior prevalência em mulheres do que em homens na impactação de caninos inferiores do lado direito, perto ou na região de linha média mandibular, porém, discorda quanto o posicionamento dentário por estar em posição vertical, e não horizontalizado como preconizam os autores.( VUCHKOVA, J et al. 2010)

No específico caso retratado neste trabalho, o tracionamento ortodôntico foi desconsiderado pelo fato de que o canino impactado estava em uma área muito próxima dos ápices dentários dos dentes anteriores inferiores e que estava na área de linha média. Esse ultimo ponto reportado desperta o fato de que a distância somada com a densidade óssea no local tornasse inviável o tracionamento REVISTA FLUMINENSE DE ODONTOLOGIA - ANO XXVII - No 55 - Janeiro / Julho 2020 
ortodôntico. Portando, optou-se pela exodontia, compactuando com( VUCHKOVA, J ET AL. 2010).

Além do tracionamento ortodôntico e a exérese do canino impactado, existe outros métodos de tratamento para os caninos inferiores impactados. Um deles é o transplante autógeno, sendo a movimentação cirúrgica do dente (vital ou tratado endodonticamente) do local original em que se encontrava para uma outra cavidade, sendo dependente de fatores como rizogêneze imcompleta, idade do paciente e garau de inclusão, o que impossibilitou no caso estudado pelo fato do canino impactado ter rizogênese completa . E o outro método é a observação radiográfica do dente impactado, que também foi descartado esse método no caso reportado pelo fato da proximidade do dente 43 nos ápices dentários e grande chance de reabsorção radicular dos incisivos centrais inferiores( CANDEIRO, G. et al 2009).

A importância dos exames de imagens auxiliares é crítica, de modo que sem eles o insucesso do planejamento cirúrgico é eminente. É importante destacar as radiografias periapicais (utilizando a regra de Clark), panorâmica e oclusal, que serviram de pilar no planejamento cirúrgico e no ato da cirurgia em sí do caso relatado (JOSHI, M.R 2001).

Um estudo feito demonstrou que a tomografia computadorizada cone beam possibilita um diagnóstico e planejamento mais preciso, principalmente em casos onde há a possibilidade de provocar reabsorções radiculares nos dentes adjacentes. Para o caso estudado, não foi necessária a realização da tomografia computadorizada pelo fato das imagens radiográficas fornecerem um bom exame auxiliar de imagem, em que foi possível localizar a posição anatômica em que o mesmo se encontrava (LUDLOW JB,et al. 2003).

O bloqueio bilateral do nervo mandibular foi feito de acordo com a técnica cirúrgica descrita, e o acesso cirúrgico intra oral com osteotomia e odontossecção para remover o dente(WERTZ RA. 1994).

\section{CONCLUSÃO}

A não erupção de um canino incluso na mandíbula deve sempre ser investigada, de modo que as imagens radiográficas são o primeiro passo para o diagnóstico dessa possível impactação. O caso deste trabalho foi conduzido de maneira adequada, visto suas características condizerem com o tipo de tratamento indicado nas literaturas de referência e executado de maneira fiel aos estudos feitos anteriormente citados neste relato. Os métodos de diagnóstico foram suficientes para o diagnóstico e planejamento cirúrgico, sendo sempre o menos REVISTA FLUMINENSE DE ODONTOLOGIA - ANO XXVII - No 55 - Janeiro / Julho 2020 
invasivo, dentro possível, com o menor uso de radiação proporcionado para uma visualização satisfatória do elemento dentário incluso.

\section{REFERÊNCIAS BIBLIOGRÁFICAS}

1. PETERSON L. Cirurgia Oral e Maxilofacial Contemporânea. Trad., $3^{\mathrm{a}}$ Ed, Rio de Janeiro: Guanabara-Koogan; 2000. 772p

2. NEVILlE BW, DMM DD, ALLEN CM, BOUQUOT JE. Patologia Oral e Maxilofacial. Trad., $2^{a}$ Ed, Rio de Janeiro: Guanabara-Koogan; 2004. 798p.

3. THILANDER, B.; MYRBERG N. The prevalence of malocclusion in Swedish school children. Scand. J. Dent. Res., Copenhagen, v. 81, no. 1, p. 12-20, 1973.

4. ROHRER, A. Displaced and impacted canines. Int. J. Orthod. Dent. Child., [S.1.], v. 15, p. 1003-1020, 1929.

5. BISHARA, S.E. Impacted maxillary canines: a review. Am. J. Orthod. Dentofacial. Orthop., St. Louis, v. 101, no. 2, p. 159-171, Feb. 1992.

6. CANDEIRO, G.; TAVARES, R. Surgical Treatament of impacted lower Canine Transmigration - case report. Rev. Fac. Odontol. Porto Alegre, v. 50, n. 3, p. 36-39, set./dez., 2009.

7. LUDLOW JB, DAVIES-LUDLOW LE, BROOKS SL. Dosimetry of two extraoral direct digital imaging devices: NewTom cone beam CT and Orthophos Plus DS panoramic unit. Dentomaxillofac Radiol. 2003;32:229-34.

8. JOSHI, M.R. Transmigrant mandibular canines: a record of 28 cases and retrospective review of the literature. Angle Orthod., Appleton, Wis., v. 71, no. 1, p. 12-22, Feb. 2001.

9. VUCHKOVA, J.; FARAH, C. S. Canine transmigration: comprehensive literature review and report of 4 new australian cases. Oral Surg. Oral Med. Oral Pathol. Oral Radiol. Endod., St. Louis, v. 109, no. 4, p. e46-e53, Apr. 2010.

10. WERTZ RA. Treatment of transmigrated mandibular canines. Am J Orthod Dentofacial Orthop. 1994; 106: 419-27. 